10. Pollyea DA, Pratz KW, Jonas BA, et al. Venetoclax in Combination with Hypomethylating Agents Induces Rapid, Deep, and Durable Responses in Patients with AML Ineligible for Intensive Therapy. Blood. 2018;132(Suppl 1):285-285.

11. DiNardo CD, Pratz KW, Letai A, et al. Safety and preliminary efficacy of venetoclax with decitabine or azacitidine in elderly patients with previously untreated acute myeloid leukaemia: a non-randomised, open-label, phase 1b study. Lancet Oncol. 2018;19(2):216228 .

12. Kubasch AS, Wehner R, Bazzurri S, et al. Clinical, molecular, and immunological responses to pembrolizumab treatment of synchronous melanoma and acute myeloid leukemia. Blood Adv. 2018;2(11):1187-1190.

13. Daver N, Garcia-Manero G, Basu S, et al. Efficacy, Safety, and Biomarkers of Response to Azacitidine and Nivolumab in Relapsed/Refractory Acute Myeloid Leukemia: A Nonrandomized, Open-Label, Phase II Study. Cancer Discov. 2019;9(3):370-383.

14. Nand S, Othus M, Godwin JE, et al. A phase 2 trial of azacitidine and gemtuzumab ozogamicin therapy in older patients with acute myeloid leukemia. Blood, 2013;122(20):3432-3439.

15. Daver N, Kantarian H, Ravandi F, et al. A phase II study of decitabine and gemtuzumab ozogamicin in newly diagnosed and relapsed acute myeloid leukemia and high-risk myelodysplastic syndrome. Leukemia. 2016;30(2):268-273.

16. Garcia-Manero G, Fenaux P, Al-Kali A, et al; ONTIME study investigators. Rigosertib ver- sus best supportive care for patients with high- risk myelodysplastic syndromes after failure of hypomethylating drugs (ONTIME): a rando- mised, controlled, phase 3 trial Lancet Oncol. 2016;17(4):496-508.

17. Navada SC, Garcia-Manero G, Atallah EL, et al. Phase 2 Expansion Study of Oral Rigosertib Combined with Azacitidine (AZA) in Patients (Pts) with Higher-Risk (HR) Myelodysplastic Syndromes (MDS) Efficacy and Safety Results in HMA Treatment Naïve \& Relapsed (Rel)/Refractory (Ref) Patients. Blood. 2018;132(Suppl 1):230.

18. Medyouf $\mathrm{H}$. The microenvironment in human myeloid malignan cies: emerging concepts and therapeutic implications. Blood. 2017;129(12): 1617-1626.

19. Stein EM, DiNardo CD, Pollyea DA, et al. Enasidenib in mutant IDH2 relapsed or refractory acute myeloid leukemia. Blood. 2017;130(6):722-731.

20. Cortes J, Perl AE, Döhner H, et al. Quizartinib, an FLT3 inhibitor, as monotherapy in patients with relapsed or refractory acute myeloid leukaemia: an open-label, multicentre, single-arm, phase 2 trial Lancet Oncol. 2018;19(7):889-903.

\title{
Discontinuation of tyrosine kinase inhibitors in patients with chronic myelogeneous leukemia - You can do this at home if you read the instructions
}

\section{Charles A. Schiffer}

Joseph Dresner Chair for Hematologic Malignancies, Departments of Oncology and Medicine, Wayne State University School of Medicine, Karmanos Cancer Institute, Detroit, MI, USA

E-mail: CHARLES A. SCHIFFER - Schiffer@karmanos.org

doi:10.3324/haematol.2019.222216

T he exciting story of the clinical use of imatinib mesylate for the treatment of leukemias driven by the bcr/abl mutation began in the late 1990s and dramatic effectiveness was immediately apparent in all stages of the diseases. Although there was concern that these benefits might not persist, we now know, after almost twenty years of follow up, that a high proportion of chronic phase patients attain deep molecular responses and enjoy an overall survival comparable to that of agematched controls. ${ }^{1}$ It was originally expected that lifelong treatment would be needed, but in recent years, trials from around the world have shown that tyrosine kinase inhibitors (TKI) can be successfully discontinued in some patients who have achieved sustained deep molecular responses. ${ }^{2,3}$

These were conducted as part of clinical trials at CML research institutions by experienced CML clinicians. In this issue of the Journal, Italian clinicians from a wide range of institutions of the Gruppo Italiano Malattie Ematologiche dell'Adulto (GIMEMA) describe a large group of chronic phase patients who had therapy discontinued, many presumably as a consequence of patients' requests to doctors, who were now comfortable with the accumulated results. ${ }^{4}$ With a median follow up of 34 months, $60 \%$ of patients remained in what has been termed "treatment-free remission" (TFR), ${ }^{5}$ a result consistent with or perhaps slightly superior to those from earlier trials. As in other trials, the relapse rate was somewhat lower in patients with longer exposures to TKI and all patients who had molecular relapse were successfully retreated with either their original TKI or were switched to another TKI if their motivation for discontinuation was toxicity; these retreated patients usually achieved the level of their original response.

Most CML patients in the US (and to some extent elsewhere) are not followed in specialty hematology centers. This means that the next question in the TKI saga is whether discontinuation can be managed safely by nonspecialist oncologists. The process is not very difficult to understand and there are few risks if patients are selected and followed appropriately. The criteria for study entry and monitoring differed somewhat amongst the published trials, but a consensus approximation would include:

- TKI treatment for a minimum of three years;

- continuous deep molecular response [minimum MR4 (BCR-ABL1 $\leq 0.01 \%$ using the International Scale, IS)] on multiple testing for at least two years. Some studies required reduction to $<M R 4.5$, although outcomes seem comparable (including the results from this GIMEMA experience) using either molecular cutoff;

- use of a quantitative polymerase chain reaction (qPCR) test sensitive to a level of at least MR4.5 in a laboratory with a rapid turn-around time;

- monitoring of peripheral blood transcripts every 4-6 weeks for 6-8 months, then bimonthly for approximately one year followed by every three months thereafter for a minimal follow up of three years; 
"Relapse" is defined by loss of MR3 [major molecular response $(M M R)]$, and it is essential to be aware that values can sometimes fluctuate between MR3 and MR4, in part because of the variability of the assay; therefore, at least two values with loss of MMR should be documented before therapy is resumed. This is perhaps the part of the process with the most subtleties, and consultation with CML specialists is sometimes advisable.

The pattern of relapse raises interesting issues about CML biology. Remarkably, despite the inclusion of patients with continuously undetectable transcripts for many years using very sensitive techniques, molecular relapses can be detected within the first 1-2 months of discontinuation. Virtually all relapses occur within the first 6-8 months of cessation, with very few emerging with long-term follow up which is now, in many studies, over five years. The rapidity of relapse in patients attests to the resilience of dormant CML progenitors which are capable of re-emerging almost immediately after the suppressive pressure of the TKI is released; it is a humbling reminder of the difficulties to be faced in eliminating stem cells in other leukemias.

Perhaps even more fascinating is the observation of prolonged remissions lasting for many years. While it is theoretically possible that CML "stem" cells have been eliminated (Figure 1), this would seem unlikely. I am not aware of any studies of bone marrows from patients in long-term TFR evaluating whether bcr/abl positive colonies can be grown in vitro. Interest has been shown in the possibility of immune suppression of remaining bcr/abl precursors, with some focus on the role of T-natural killer (NK) cells, ${ }^{6,7}$ perhaps stimulated by observations of a possible salutary effect of proliferation of NK cells after dasatinib treatment. ${ }^{8,9}$ Results of these studies have been, at best, inconclusive.

Nonetheless, this remains an interesting hypothesis. I have seen two patients in apparent TFR who experienced molecular relapse after 1-2 years of follow up: the first after chemotherapy for another cancer and the other after prolonged use of corticosteroids for treatment of the TKI "withdrawal" syndrome. Could the relapses have been related to "immunosuppression" from these other treatments? This is speculative at best, but it would be interesting to see if other such patients are identified. Changes in the marrow microenvironment might also play a role in either the continued containment of growth or, alternatively, promote rapid recurrence.

It is also not clear whether late relapses will develop with longer follow up. As illustrated in Figure 1, it is possible that successful TKI therapy reduces the number of CML precursors to the levels found in 'preclinical' CML. Little is known about the duration of the 'incubation' period after the initial mutagenic event or how long it takes for CML to become clinically identifiable. Perhaps

\section{Diagnosis}

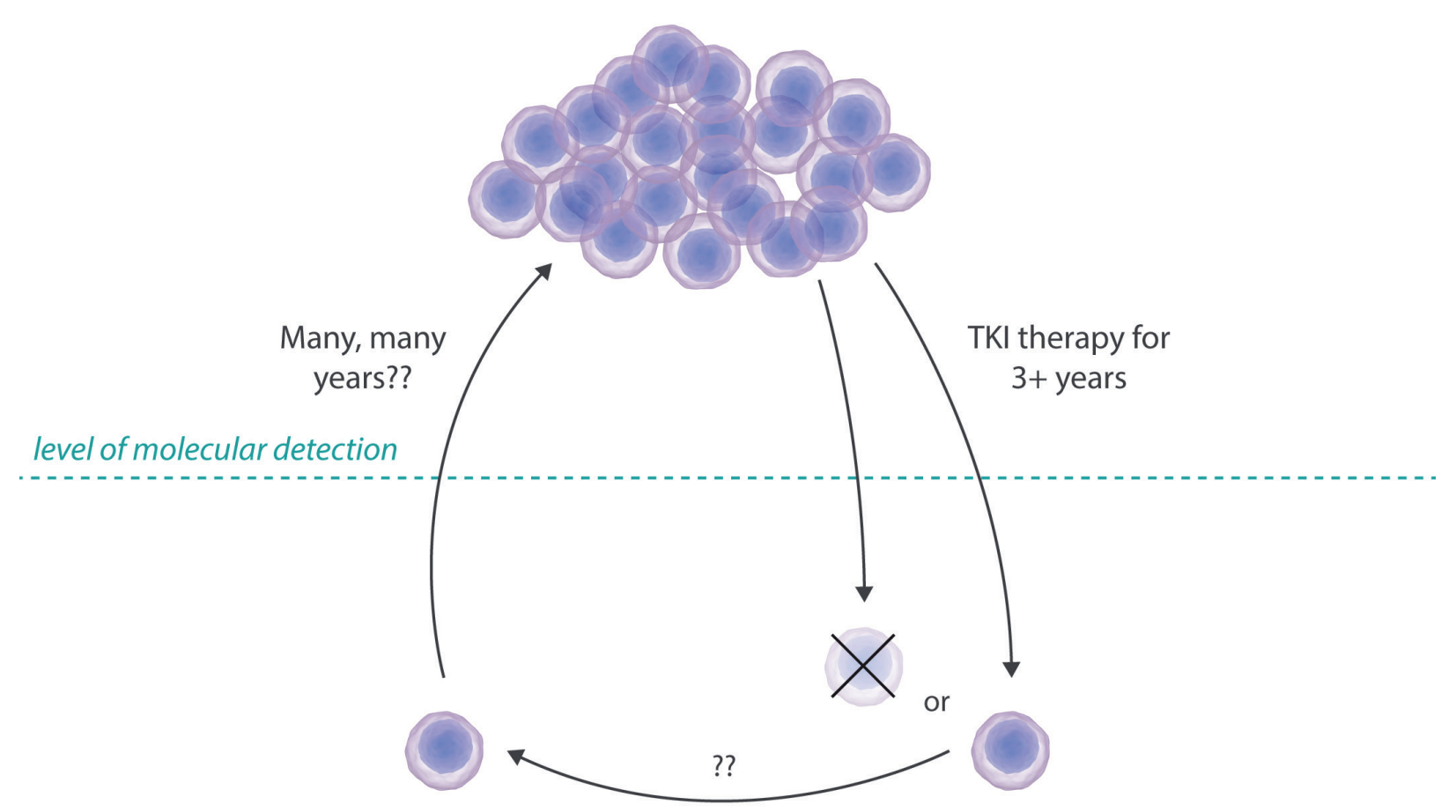

\section{Preclinical}

Figure 1. Chronic myeloid leukemia (CML) has a preclinical phase of unknown duration and usually is diagnosed with obvious "bulk" myeloid proliferation. After tyrosine kinase inhibitor (TKI) treatment, the cellular burden is markedly reduced to below the level of molecular detection (horizontal line). It is possible that the $\mathrm{CML}$ precursors are entirely eliminated $(\mathrm{X})$ or tiny amounts of residual disease persist which have the potential to become clinically apparent after a long period of time, analogous to what occurs in the original presentation of the disease. 
the most relevant data come from observations after the atomic bomb events in Japan where the incidence of CML peaked at a median of ten years but continued at an increased rate for years thereafter. ${ }^{10}$ These findings have implications about the frequency or even the necessity of long-term PCR monitoring of patients in long-term remission. I have adopted a non-data-driven approach and continue testing approximately every six months after three years of undetectable transcripts. However, we urgently need further information about this.

It is also important to appreciate that only a minority of chronic phase patients can achieve long-term TFR. Most treatment trials describe the rates of molecular response using cumulative incidence analyses, meaning that a patient achieved that level of response at least once. It is more difficult, however, to identify the rates of sustained response, a requirement for considering stopping. In the imatinib-based German CML IV and IRIS trials (the two largest studies with long-term follow up), the rate of MR 4.5 was approximately $50 \%$ at five years and $>60 \%$ at ten years, respectively, although these estimates were not based on the "intent to treat" population ${ }^{11,12}$ and only included patients for whom data were available at these time points.

Using a somewhat generous estimate of $40 \%$ sustained MR4.5 in newly diagnosed chronic phase patients, and a relapse rate of $50 \%$, only approximately $20 \%$ of patients will successfully achieve TFR. Calculations may differ somewhat using continuous MR4 as the eligibility cutoff or if patients were treated initially with second-generation TKI which produce higher response rates. Nonetheless, the reality is that the large majority of patients will require life-long treatment, and even those who stop successfully would have required many years of treatment prior to a trial of cessation. Therefore, optimal CML treatment will continue to depend on the skills of physicians familiar with ameliorating the side effects of therapy and health systems that deal more effectively with the costs of this chronic treatment.

And this raises the question of whether all patients require the "standard" dose to maintain response or if many of the benefits of stopping, such as reduced side effects and costs, can be achieved with lower doses. Again, data are fragmentary, but many clinical trials and observational studies report that a significant proportion of patients are maintained long term on lower than the initial "standard" doses of TKI. ${ }^{13,14}$ A recent pilot trial in newly diagnosed patients demonstrated what appear to be identical response rates with less toxicity, using $50 \mathrm{mg}$ of dasatinib versus the standard $100 \mathrm{mg}$ dose. ${ }^{15}$ Indeed, based on these data, I have been decreasing the dasatinib dose to $50 \mathrm{mg}$ in patients with stable high-grade responses on $100 \mathrm{mg}$ with no evidence of loss of benefit in approximately a dozen patients. ${ }^{16}$

Perhaps the most systematic data come from the UK DESTINY trial in which patients eligible for consideration for a TFR trial had their doses reduced by $50 \%$ for one year before drug discontinuation. ${ }^{17}$ Two per cent of patients who entered the trial with levels of MR4 lost MMR during the first year, while only $18 \%$ of those who began with sustained MR3 lost MMR within the year. It is not known how patients would have fared long term on the lower dose since they went on to the TFR portion of the study. Those who "relapsed" within the first year had therapy restarted, but it is possible that that may not have always been necessary. In fact, a recent modeling exercise using data from large clinical trials suggests that the rise in transcript numbers after dose reduction can be transient in many patients, and that MMR response might have recovered without increasing the dose. ${ }^{18}$ It is, therefore, clear that a substantial number of patients can do well with lower doses of TKI, but prospective trials addressing this question would be welcomed.

To conclude, the report from the GIMEMA group confirms that TFR can be achieved in routine clinical practice and indicates that discontinuation be considered in appropriately selected patients outside the clinical trial setting. ${ }^{4}$ The relapse rate has been consistently in the $50 \%$ range in all trials, and future research should focus on the mechanisms by which recurrence is suppressed in the hope that new approaches, possibly immunomodulatory, can improve these results. In addition, patients should continue to be monitored to assess whether very late relapses develop.

\section{References}

1. Bower H, Björkholm M, Dickman PW, et al. Life expectancy of patients with chronic myeloid leukemia approaches the life expectancy of the general population. J Clin Oncol. 2016;34(24):2851-2857.

2. Etienne G, Guilhot I, Rea D, Rigal-Huguet F, et al. Long-Term Follow-Up of the French Stop Imatinib (STIM1) Study in Patients With Chronic Myeloid Leukemia. J Clin Oncol. 2017;35(3):298-305.

3. Saussele S, Richter J, Guilhot I, et al. Discontinuation of tyrosine kinase inhibitor therapy in chronic myeloid leukaemia (EURO-SKI): a prespecified interim analysis of a prospective, multicentre, nonrandomised, trial. Lancet Oncol. 2018;19(6):747-757.

4. Fava C, Rege-Cambrin G, Dogliotti I, et al. Observational Study of CML Chronic Myeloid Leukemia Italian patients who discontinued Tyrosine Kinase Inhibitors in clinical practice. Haematologica. 2019; 104(8):1589-1596.

5. Hughes TP, Ross DM. Moving treatment-free remission into mainstream clinical practice in CML. Blood. 2016;128(1):17-23.

6. Ilander $\mathrm{M}$, Olsson-Strömberg U, Schlums $\mathrm{H}$, et al. Increased proportion of mature NK cells is associated with successful imatinib discontinuation in chronic myeloid leukemia. Leukemia. 2017;31(5):11081116

7. Réa D, Henry G, Khaznadar Z, et al. Natural killer-cell counts are associated with molecular relapse-free survival after imatinib discontinuation in chronic myeloid leukemia: the IMMUNOSTIM study. Haematologica. 2017;102(8):1368-1377.

8. Mustioki S1, Ekblom M, Arstila TP, et al. Clonal expansion of T/NKcells during tyrosine kinase inhibitor dasatinib therapy. Leukemia. 2009;23(8):1398-1405.

9. Schiffer CA, Cortes I, Hochhaus A, et al. Lymphocytosis following treatment with dasatinib in chronic myeloid leukemia: effects on response and toxicity. Cancer. 2016;122(9):1398-1407.

10. Hsu WL, Preston DL, Soda M, et al. The Incidence of Leukemia, Lymphoma and Multiple Myeloma among Atomic Bomb Survivors: 1950-2001. Radiat Res. 2013;179(3):361-382.

11. Hehlmann $R$, Lauseker $M$, Saußele $S$, et al. Assessment of imatinib as first-line treatment of chronic myeloid leukemia: 10-year survival results of the randomized CML study IV and impact of non-CML determinants. Leukemia. 2017;31(11):2398-2406.

12. Hochhaus A, Larson RA, Guilhot F, et al. Long-Term Outcomes of Imatinib Treatment for Chronic Myeloid Leukemia. N Engl J Med. 2017;376(10):917-927.

13. Faber E, Divoká M, Skoumalová I, et al. A lower dosage of imatinib is sufficient to maintain undetectable disease in patients with chronic myeloid leukemia with long-term low-grade toxicity of the treatment. Leuk Lymphoma. 2016;57(2):370-375.

14. Visani G, Breccia M, Gozzini A, et al. Dasatinib, even at low doses, is an effective second-line therapy for chronic myeloid leukemia 
patients resistant or intolerant to imatinib. Results from a real lifebased Italian multicenter retrospective study on 114 patients. Am J Hematol. 2010;85(12):960-963.

15. Clark RE, Polydoros F, Apperley JF, et al. De-escalation of tyrosine kinase inhibitor dose in patients with chronic myeloid leukaemia with stable major molecular response (DESTINY): an interim analysis of a non-randomised, phase 2 trial. Lancet Haematol. 2017;4(7):e310-e316.

16. Naqvi K, Jabbour E, Skinner J, et al. Early results of lower dose dasa- tinib (50 mg daily) as frontline therapy for newly diagnosed chronicphase chronic myeloid leukemia. Cancer. 2018;124(13):2740-2747.

17. Schiffer CA. The evolution of dasatinib dosage over the years and its relevance to other anticancer medications. Cancer. 2018;124(13): 2687-2689.

18. Fassoni AC, Baldow C, Roeder I, Glauche I. Reduced tyrosine kinase inhibitor dose is predicted to be as effective as standard dose in chronic myeloid leukemia: a simulation study based on phase III trial data. Haematologica. 2018;103(11):1825-1834.

\section{The only thing that stops a bad microbiome, is a good microbiome}

\section{Jessica R. Galloway-Peñ $a^{1,2}$ and Robert R. Jen $q^{1,3}$}

${ }^{1}$ Department of Genomic Medicine; ${ }^{2}$ Department of Infectious Diseases, Infection Control and Employee Health and ${ }^{3}$ Department of Stem Cell Transplantation, The University of Texas MD Anderson Cancer Center, Houston, TX, USA.

E-mail:jrgalloway@mdanderson.org/rrjenq@mdanderson.org

doi:10.3324/haematol.2019.222430

M ultidrug resistant (MDR) bacterial colonization in the gut is frequently induced by excessive use of antibiotics. ${ }^{1}$ Fecal microbiota transplantation (FMT) has been shown to be quite successful in treating refractory and recurrent Clostridium difficile infection. ${ }^{2}$ Thus, current research is focusing on how FMT may also help in decolonizing MDR organisms (MDRO) and in preventing recurrent $\mathrm{MDR}$ infections. ${ }^{3}$ Decolonization of MDRO via FMT may be particularly useful in patients with hematologic malignancies, such as those undergoing hematopoietic stem cell transplantation (HSCT), ${ }^{4}$ as use of chemotherapeutic agents and frequent administration of antibiotics can favor the selection of resistant pathogens. ${ }^{5,6}$

In spite of the increasing evidence that the feasibility and safety of FMT in immunocompromised cohorts is comparable to that of immunocompetent patients, administering FMT in the setting of hematologic malignancy remains a cause for concern due to perceived risks of translocation and sepsis. ${ }^{7,8}$ Given the growing body of literature associating a dysbiotic microbiome with adverse HSCT outcomes and treatment-related toxicities, including infection, delivering a diverse microbiome via FMT to immunocompromised patients may provide a variety of benefits, such as promoting colonization resistance and reducing the risk of bacterial translocation. Thus, attempts to better characterize the safety and efficacy of FMT in these patients are merited.

In this issue of the Journal, Battipaglia et al. ${ }^{10}$ describe a retrospective case series of 10 patients with hematologic malignancies undergoing FMT for MDRO colonization before or after allogeneic HSCT. In this study, the authors show both the safety and efficacy of using FMT for decolonization of carbapenem-resistant Enterobactericeae (CRE), carbapenem-resistant Pseudomonas (CRP), and vancomycin-resistant Enterococcus (VRE). Notably, the study reports FMT both pre- and post transplant. The majority of patients who received FMT prior to transplant did not have recurrent MDRO even after HSCT, indicating the prophylactic use of FMT. Interestingly, the procedure remained effective for long- term MDRO decolonization in the majority of patients despite the use of broad-spectrum antibiotics in some of the patients after FMT. This implies that FMT can potentially achieve full decolonization of MDRO rather than merely reducing the levels of MDRO below the limit of detection.

While FMT was shown to be successful in decolonizing the MDRO studied, the FMT did not always prevent additional post-transplant infections from other bacteria susceptible to antibiotics. Interestingly, only $50 \%$ of patients concomitantly colonized with extended spectrum $\beta$-lactamase (ESBL)-producing Enterobacteriaceae obtained decolonization. This is reminiscent of a case report by Stalenhoef et al. where FMT successfully eradicated a Pseudomonas aeruginosa urinary tract infection, while stool cultures remained positive for extended-spectrum $\beta$-lactamase (ESBL)-producing Enterobacteriaceae three months after FMT. ${ }^{11}$ Thus, the higher frequency of failure of FMT to eradicate the ESBL-producing Enterobacteriaceae in these two studies may suggest potential limitations to this therapy.

Although the specific mechanisms underlying the success of FMT for MDRO colonization remain unclear, Figure 1 depicts an overview of the general concepts regarding the use of FMT for MDRO in patients with hematologic malignancies. Given that this study looked specifically at CRE, CRP, and VRE, it remains unclear if other MDRO may be equally responsive to FMT. Furthermore, given the seemingly discrepant results for CRE, CRP, and VRE compared to ESBL-producing organisms, one might consider that distinct mechanisms of action underlie how FMT mediates response for different MDRO.

Due to the retrospective nature of the study, in contrast to a controlled study, it is unclear how physicians decided to treat each patient with FMT case by case. Moreover, there was a large variation between cases in the time of FMT relative to HSCT and the MDRO colonization/infection in both pre- and post-HSCT groups. Thus, it remains to be determined what the ideal timeframe for FMT is in both scenarios. The use of relat- 